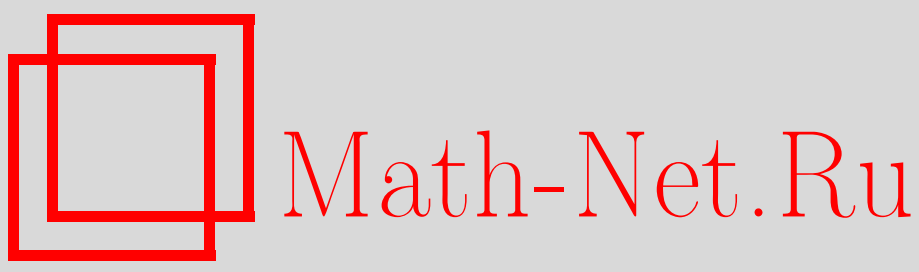

Е. В. Жужома, В. С. Медведев, О неориентируемых двумерных базисных множествах на 3-многообразиях, Матем. сб., 2002, том 193, номер 6, 83-104

DOI: https://doi.org/10.4213/sm661

Использование Общероссийского математического портала Math-Net.Ru подразумевает, что вы прочитали и согласны с пользовательским соглашением

http://www . mathnet.ru/rus/agreement

Параметры загрузки:

IP : 54.205 .225 .156

26 апреля 2023 г., 15:33:44 
УДК $517.917+513.9$

\author{
Е.В. Жужома, В.С. Медведев
}

\title{
О неориентируемых двумерных базисных множествах на 3-многообразиях
}

\begin{abstract}
В статье доказано, что структурно устойчивьй диффеоморфизм $f: M^{3} \rightarrow M^{3}$ замкнутого трехмерного многообразия $M^{3}$ не содержит в спектральном разложении неориентируемых растягивающихся аттракторов и сжимающихся репеллеров коразмерности один.

Библиография: 36 названий.
\end{abstract}

\section{Введение}

Важным и достаточно хорошо изученным классом структурно устойчивых динамических систем являются диффеоморфизмы Аносова коразмерности один [1]-[4]. Известно, что любой такой диффеоморфизм топологически сопряжен гиперболическому автоморфизму тора и, кроме того, два таких диффеоморфизма топологически сопряжены тогда и только тогда, когда они $\pi_{1}$-сопряжены [5], [6] (последнее означает, что они индуцируют сопряженные изоморфизмы фундаментальной группы тора). Тор $T^{n}$ является единственным базисным множеством топологической размерности $n=\operatorname{dim} T^{n} \geqslant 2$.

Непосредственным обобщением диффеоморфизмов Аносова являются структурно устойчивые $A$-диффеоморфизмы, введенные Смейлом [7]. Если к диффеоморфизму Аносова коразмерности один применить “хирургическую” операцию, то получим структурно устойчивый $A$-диффеоморфизм с нетривиальным базисным множеством коразмерности один, локально гомеоморфным произведению гиперплоскости коразмерности один на канторовское множество [7]. В зависимости от исходного диффеоморфизма Аносова полученное нетривиальное базисное множество $\Omega$ является либо растягивающимся аттрактором, либо сжимающимся репеллером. При этом достижимая из $T^{n}-\Omega$ граница любой компоненты множества $T^{n}-\Omega$ состоит из двух инвариантных многообразий коразмерности один, так называемой 2-связки. Следуя [7], будем называть полученньй $A$-диффеоморфизм $D A$-диффеоморфизмом. Отметим, что $D A$-диффеоморфизм имеет ориентируемое базисное множество $\Omega$ и может быть построен на торе $T^{n}$ любой размерности $n \geqslant 2$.

Имеется несколько определений ориентируемости базисного множества, два из которых наиболее употребимы. Одно определение дается через ориентируемость соответствующих подрасслоений касательного расслоения (см., например, [8], [9]).

Работа вьполнена при поддержке Российского фонда фундаментальных исследований (гранты № № 99-01-00230, 02-01-000-98) и форонда INTAS (грант № 97-1843).

(C) Е. В. Жужома, В. С. Медведев 2002 
Другое, введенное Гринесом [10], использует индекс пересечений инвариантных многообразий (см. определение в $\S 1$ ). Мы будем под ориентируемостью базисного множества понимать ориентируемость в смысле Гринеса.

Первый пример неориентируемого базисного множества коразмерности один, которое является растягивающимся аттрактором или сжимающимся репеллером, был построен Плькиным [11] на двумерной сфере $S^{2}$ (следовательно, в примере Плыкина базисное множество коразмерности один является одномерным). Отметим также, что диффеоморфизм в этом примере структурно устойчивый. В дальнейшем неориентируемые одномерные базисные множества на двумерных замкнутых многообразиях исследовались в работах [12]-[15] (см. также комбинаторное описание в [16], [17]). Основной особенностью растягивающегося аттрактора $\Omega$ Плыкина [11] является наличие компоненты множества $S^{2}-\Omega$, достижимая из $S^{2}-\Omega$ граница которой состоит ровно из одного неустойчивого одномерного инвариантного многообразия, так называемой 1-связки (см. §1). Учитывая этот факт, будем называть базисное множество коразмерности один, которое является либо растягиваюшимся аттрактором, либо сжимаюшимся репеллером, базисным множеством Плыкина, если это базисное множество имеет 1-связки. Нетрудно видеть, что базисное множество Плькина неориентируемо в смысле Гринеса.

Согласно теореме 1 из [18] базисное множество коразмерности один является либо аттрактором, либо репеллером. Если оно не образует вложенное компактное подмногообразие коразмерности один, то остается открытым вопрос (насколько известно авторам), имеются ли другие базисные множества топологической размерности $n-1$, отличные от растягивающихся аттракторов или сжимающихся репеллеров (на компактных $n$-мерных многообразиях при $n \geqslant 3$ ). В настоящей статье мы рассматриваем неориентируемые растягивающиеся аттракторы и сжимающиеся репеллеры коразмерности один. Известно, что эти базисные множества локально гомеоморфны произведению гиперплоскости коразмерности один на канторовское множество. Так как утверждения, касаюшиеся аттракторов, легко переформулировать для репеллеров, то в дальнейшем мы в основном будем говорить об аттракторах.

Приведем некоторые результаты, относящиеся к данной тематике. В [15] приводится конструкция примеров (в теореме о реализации) неориентируемых растягивающихся аттракторов коразмерности один на открытых $n$-мерных, $n \geqslant 3$, многообразиях и получен полньй инвариант топологической сопряженности ограничения $A$-диффеоморфизма на некоторую притягиваюшую область аттрактора. Отметим еще две работы [8] и [9], в которых ориентируемость базисного множества понимается как ориентируемость устойчивого и неустойчивого подрасслоений касательного расслоения над базисньм множеством. В [8] доказано, что несущее многообразие с базисным множеством коразмерности один должно иметь нетривиальную фундаментальную группу. В [9] показывается, что ориентируемое базисное множество коразмерности один определяет ненулевой элемент одномерной групшы гомологий (в частности, несущее многообразие имеет нетривиальную одномерную группу гомологий). Оба этих результата уточнены в [15], где доказано, что фундаментальная группа несушего многообразия содержит подгруппу, изоморфную $n$-мерной целочисленной решетке. 
Цель настоящей статьи - исследовать вопрос о существовании неориентируемых растягивающихся аттракторов и сжимающихся репеллеров коразмерности один (и, в частности, базисных множеств Плькина) у структурно устойчивых диффеоморфизмов на замкнутых трехмерных многообразиях. Основной результат статьи содержится в следуюшей теореме (см. §3).

Теорема 3.3. Пусть $f: M^{3} \rightarrow M^{3}$ - структурно устойчивъй диффеоморфизм замкнутого трехмерного многообразия $M^{3}$. Тогда спектральное разложсение диффеоморфизма $f$ не содержит неориентируемых растягивающихся аттракторов и сжимающихся репеллеров коразмерности один.

СлЕДСТВИЕ 3.4. Пусть $f: M^{3} \rightarrow M^{3}$ - структурно устойчивый диффеоморфизм замкнутого трехмерного многообразия $M^{3}$. Тогда $f$ не имеет базисных мно жеств Плькина.

Упоминаемый пример Плыкина [11] показывает, что теорема 3.3 и следствие 3.4 неверны в размерности $n=2$. Теорема 3.3 , по сушеству, вытекает из теоремы 3.1 , в которой утверждается, что если растягивающийся аттрактор коразмерности один имеет 1-связку, то многообразие $M^{3}$ неориентируемое, и теоремы 3.2 , в которой показывается, что растягивающийся аттрактор коразмерности один структурно устойчивого диффеоморфизма не имеет 1-связок. Далеемы используем лемму 2.5 о том, что растягиваюшийся аттрактор коразмерности один ориентируем (в смысле Гринеса) тогда и только тогда, когда все его связки имеют степень два. Последнее утверждение составляет часть теоремы 2.1, анонсированной в [15]. Насколько нам известно, доказательство этой теоремы в доступном издании пока не опубликовано. Поэтому для удобства читателя мы приводим доказательство этого утверждения, для простоты потребовав дополнительно наличие структурной устойчивости диффеоморфизма.

После доказательства теоремы 3.3 естественно возникает вопрос о существовании неориентируемых растягивающихся аттракторов и сжимающихся репеллеров (в частности, базисных множеств Плыкина) коразмерности один на замкнутых 3 -многообразиях. В $\S 3$ мы строим пример $\Omega$-устойчивого (но структурно неустойчивого в силу теоремы 3.3$) A$-диффеоморфизма 3 -мерного замкнутого многообразия, который имеет неориентируемьй растягивающийся аттрактор $\Omega_{1}$ и сжимаюшийся репеллер $\Omega_{2}$ коразмерности один. Это доставляет очередной пример существования области в пространстве диффеоморфизмов, заполненной структурно неустойчивыми диффеоморфизмами.

Для размерности $n \geqslant 3$ мы уточняем результат работы [8]. Именно доказывается следующая теорема.

ТЕОРемА 3.4. Пусть $\Omega$ - растягивающийся аттрактор коразмерности один $A$-диффеоморфизма $f: M^{n} \rightarrow M^{n}$ компактного многообразия $M^{n}$ размерности $n \geqslant 3$. Тогда каждая компонента множсества $M^{n}-\Omega$, в достижимую граничу которой входит 1-связка, имеет нетривиальную фундаментальную группу.

Отметим, что для компоненты множества $M^{3}-\Omega$, в достижимую границу которой входит 2-связка, теорема 3.4 , вообще говоря, неверна. 
Структура статьи следующая. В $\S 1$ даются основные определения. В $\S 2$ доказываются вспомогательные результаты и, в частности, лемма 2.4 о несуществовании трансверсально неориентируемых слоений со слоями, гомеоморфньми плоскостям. Эта лемма используется для доказательства леммы 2.5. В $\S 3$ доказываются основные теоремы $3.3,3.4$, а также необходимые для их доказательства теоремы 3.1 и 3.2 .

Авторы благодарят Д.В. Аносова, С. Х. Арансона, В.З. Гринеса за плодотворные обсуждения. Мы благодарим А. Ю. Жирова, внимательно прочитавшего рукопись и сделавшего ряд полезных замечаний. Особая благодарность М.И. Малкину - нашему “локальному” руководителю по гранту INTAS.

\section{§1. Основные определения}

Напомним некоторые понятия и факты, касающиеся диффеоморфизмов, удовлетворяюших аксиоме А. Хорошим источником являются книга [19], а также обзорные статьи [4], [20] и [7].

Пусть $f$ - гладкий $A$-диффеоморфизм замкнутого $n$-мерного $(n \geqslant 2)$ многообразия $M$. Базисное множество $\Omega$ диффеоморфизма $f$ называется аттрактором, если существует окрестность $U$ этого множества такая, что $\bigcap_{j \geqslant 0} f^{j}(U)=\Omega$. Множество назьвается репеллером, если оно является аттрактором для $f^{-1}$. Аттрактор $\Omega$ называется растягивающимся аттрактором коразмерности один, если размерность устойчивого многообразия каждой его точки равна единице, $\operatorname{dim} E_{\Omega}^{s}=1$, и $\operatorname{dim} \Omega=n-1$. Согласно [18; теорема 2] $\Omega$ состоит из $(n-1)$-мерных неустойчивых многообразий своих точек и имеет локальную структуру прямого произведения $(n-1)$-мерного евклидова пространства и канторовского множества. Базисное множество $\Omega$ называется сжимающимся репеллером, если оно является растягивающимся аттрактором для $f^{-1}$. Ясно, что многие результаты об аттракторах можно перефразировать применительно к репеллерам и наоборот.

Каждое инвариантное многообразие есть образ евклидова пространства относительно гладкой иммерсии. Поэтому оно является ориентируемьм подмногообразием (подмногообразие мы рассматриваем как подмножество такое, что отображение включения регулярно). В силу его односвязности каждое инвариантное многообразие является также нормально ориентируемьм. Поэтому корректно следуюшее определение ориентируемости базисного множества. Следуя [10], [21], будем говорить, что базисное множество $\Omega$ ориентируемо (в смысле Гринеса), если для любой точки $x \in \Omega$ и любых фиксированных чисел $\alpha>0, \beta>0$ индекс пересечения $W_{\alpha}^{s}(x) \cap W_{\beta}^{u}(x)$ во всех точках пересечения один и тот же $(+1$ либо -1$)$. В противном случае базисное множество $\Omega$ назьвается неориентируемым.

При формулировке условий структурной устойчивости большую роль играет условие, которое называют сильным условием трансверсальности. Пусть $W_{1}, W_{2} \subset M$ - два иммерсированных подмногообразия, имеющих непустое пересечение. По определению $W_{1}, W_{2}$ пересекаются трансверсально, если для любой точки $x \in W_{1} \cap W_{2}$ касательное пространство $T_{x} M$ порождается касательньми подпространствами $T_{x} W_{1}$ и $T_{x} W_{2}$. В частности, $\operatorname{dim} T_{x} W_{1}+$ $\operatorname{dim} T_{x} W_{2} \geqslant \operatorname{dim} T_{x} M$. А-диффеоморфизм удовлетворяет сильному условию 
трансверсальности, если для любых точек $x, y \in N W(f)$ многообразия $W^{s}(x), W^{u}(y)$ имеют (если пересекаются) только трансверсальные пересечения.

Известно, что диффеоморфизм структурно устойчив тогда и только тогда, когда он является $A$-диффеоморфизмом и удовлетворяет сильному условию трансверсальности. Необходимость этого утверждения доказал Мане [22], достаточность Робинсон [23].

Граничные периодические точки. Пусть $\Omega$ - растягиваюшийся аттрактор коразмерности один $A$-диффеоморфизма $f: M \rightarrow M$. Обозначим через $(x, y)^{s}$ (соответственно $[x, y]^{s}$ ) открытьй (соответственно замкнутьй) интервал на кривой $W^{s}(z)$ с концевыми точками $x, y \in W^{s}(z)$, где $z \in \Omega$.

Следуя [10], [21], будем называть точку $p \in \Omega$ әраничной, если одна из компонент множества $W^{s}(p)-p$ не пересекается с $\Omega$. Так как $\Omega \neq M$, то граничные точки существуют [6], [11]. Из работ [6], [10], [11], [15], [21] вытекают следующие свойства граничных точек:

1) имеется только конечное число граничных точек и все они периодические;

2) имеется ровно одна компонента множества $W^{s}(p)-p$, которая не пересекается с $\Omega$; эту компоненту мы будем обозначать через $W_{\varnothing}^{s}(p)$;

$3)$ если $p \in \Omega$ - граничная точка, то для любой точки $x \in W^{u}(p)-p$ сушествует единственная дуга $(x, y)^{s}$ такая, что $(x, y)^{s} \cap \Omega=\varnothing$ и $y \in \Omega$; эту дугу мы будем обозначать через $(x, y)_{\varnothing}^{s}$.

Связки базисного множества коразмерности один. Пусть $G \subset M-$ множество с границей $\partial G$. Подмножество $\delta G \subset \partial G$ границы называется достижим ий $($ из $G$ ) граниией, если для любой точки $x \in \delta G$ найдется открытая дуга, полностью лежащая в $G$, такая, что $x$ является одной из ее концевых точек.

Пусть $\Omega$ - растягивающийся аттрактор коразмерности один $A$-диффеоморфизма $f: M \rightarrow M$. В работе [15] исследована достижимая граница множества $M-\Omega$ : $\delta(M-\Omega)$ есть объединение конечного числа неустойчивых многообразий всех граничных периодических точек аттрактора $\Omega$. Эта достижимая граница разбивается на попарно непересекающиеся связки, которые определяются следующим образом: попарно различные неустойчивые многообразия $W^{u}\left(p_{1}\right), \ldots, W^{u}\left(p_{k}\right)$ образуют $k$-связкy, если существуют точки $x_{i} \in W^{u}\left(p_{i}\right)$ и дуги $\left(x_{i}, y_{i}\right)_{\varnothing}^{s}, y_{i} \in$ $W^{u}\left(p_{i+1}\right), 1 \leqslant i \leqslant k+1$, такие, что $W^{u}\left(p_{k+1}\right)=W^{u}\left(p_{1}\right)$ (таким образом, $\left.y_{k} \in W^{u}\left(p_{1}\right)\right)$, при этом число $k$ максимально возможное (другими словами, не существует $(k+1)$-связки, содержащей данную связку). Точки $p_{1}, \ldots, p_{k}$ называются ассоциированными. Из инвариантности аттрактора следует, что ассоциированные точки имеют одинаковый период, который мы будем обозначать через $m\left(p_{1}, \ldots, p_{k}\right)$.

Плькин [15] доказал, что в размерности $n=\operatorname{dim} M \geqslant 3$ возможны только 1и 2-связки. В $\S 2$ нам понадобится понятие характеристической сферы, которое вводится для 2-связки.

Характеристические сферы и связывающие цилиндры. Пусть $\Omega$ - растягивающийся аттрактор коразмерности один $A$-диффеоморфизма $f: M \rightarrow M$, и пусть $B_{p q}-2$-связка аттрактора $\Omega$, состоящая из двух неустойчивых многообразий 
$W^{u}(p)$ и $W^{u}(q)$ ассоциированных граничных периодических точек $p$ и $q$ соответственно. Как указывалось вьше, для каждой точки $x \in W^{u}(p)$ имеется единственная точка $y \in W^{u}(q)$ такая, что $(x, y)^{s}=(x, y)_{\varnothing}^{s}$, и обратно, для каждой точки $y \in W^{u}(q)$ имеется единственная точка $x \in W^{u}(p)$ с $(x, y)^{s}=(x, y)_{\varnothing}^{s}$. Определим отображение

$$
\varphi:\left(W^{u}(p)-p\right) \cup\left(W^{u}(q)-q\right) \rightarrow\left(W^{u}(p)-p\right) \cup\left(W^{u}(q)-q\right),
$$

положив $\varphi(x)=y$, где $(x, y)^{s}=(x, y)_{\varnothing}^{s}$. Тогда $($ см. лемму 2.1 в $\S 2)$

$$
\left.\varphi\right|_{W^{u}(p)-p}: W^{u}(p)-p \rightarrow W^{u}(q)-q \text { и }\left.\varphi\right|_{W^{u}(q)-q}: W^{u}(q)-q \rightarrow W^{u}(p)-p
$$

т.е. отображение $\varphi$ переводит проколотые неустойчивые многообразия 2-связки друг в друга и является инволюцией. В силу теоремы о непрерывной зависимости инвариантных многообразий от начальных условий $\varphi$ - гомеоморфизм. Из инвариантности $\Omega$ вытекает соотношение

$$
\left.f^{m n} \circ \varphi\right|_{\left(W^{u}(p)-p\right) \cup\left(W^{u}(q)-q\right)}=\left.\varphi \circ f^{m n}\right|_{\left(W^{u}(p)-p\right) \cup\left(W^{u}(q)-q\right)},
$$

где $n \in \mathbb{Z}$ и $m=m(p, q)$ - период точек $p, q$.

Так как ограничение $\left.f^{m}\right|_{W^{u}(p)}$ имеет ровно одну гиперболическую отталкиваюшую неподвижную точку $p$, то в силу теоремы Гробмана-Хартмана (см., например, [19]) существует замкнутый $(n-1)$-мерный шар $D_{p} \subset W^{u}(p)$, ограниченньй цилиндрически вложенной в $W^{u}(p)(n-2)$-мерной сферой $S_{p}^{n-2}=\partial D_{p}$, такой, что $p \in \operatorname{int} D_{p}=D_{p}-\partial D_{p}$ и $D_{p} \subset \operatorname{int} f^{m}\left(D_{p}\right)$, где int $D$ означает внутренность множества $D$. Тогда множество $C_{p q}=\bigcup_{x \in \partial D_{p}}(x, \varphi(x))^{s}$ гомеоморфно открытому $(n-1)$-мерному цилиндру $\Sigma^{n-2} \times(0,1)$. Действительно, пусть $\nu: S_{p}^{n-2} \rightarrow \Sigma^{n-2}-$ произвольный гомеоморфизм. Обозначим через $\lambda_{x}(z)$ длину отрезка $(x, z)^{s} \subset(x, y)_{\varnothing}^{s}$. В силу непрерывной зависимости инвариантных многообразий от начальных условий на компактных множествах функция $\lambda_{x}(z)$ непрерывно зависит от переменных $x$ и $z$. Из компактности $S_{p}^{n-2}$ и определения связки следует, что $\lambda_{x}(\varphi(x)) \geqslant \alpha>0$ при всех $x \in S_{p}^{n-2}$. Определим $\nu_{p q}: C_{p q} \rightarrow \Sigma^{n-2} \times(0,1)$, положив

$$
\nu_{p q}(z)=\left(\nu(x), \frac{\lambda_{x}(z)}{\lambda_{x}(\varphi(x))}\right), \quad \text { где } z \in(x, \varphi(x))^{s} .
$$

Так как устойчивые многообразия попарно не пересекаются, то $\nu_{p q}$ является взаимно однозначным отображением. Из непрерывности $\lambda_{x}(z)$ и из того, что $(n-2)-$ мерные сферы $S_{p}^{n-2}$ и $S_{q}^{n-2}=\varphi\left(S_{p}^{n-2}\right)$ находятся друг от друга на ненулевом расстоянии, вытекает, что $\nu_{p q}$ - гомеоморфизм.

Множество $C_{p q}$ мы будем называть связывающим иилиндром. Так как $\varphi-$ гомеоморфизм, то $S_{q}^{n-2}=\varphi\left(S_{p}^{n-2}\right)$ является $(n-2)$-мерной сферой, цилиндрически вложенной в $W^{u}(q)$. Согласно обобшенной теореме Шенфлиса [24; гл. 5] $S_{q}^{n-2}$ ограничивает в $W^{u}(q)(n-1)$-мерньй шар, которьй мы обозначим через $D_{q}$. 
Из равенства $\left.f^{m n} \circ \varphi\right|_{\left(W^{u}(p)-p\right) \cup\left(W^{u}(q)-q\right)}=\left.\varphi \circ f^{m n}\right|_{\left(W^{u}(p)-p\right) \cup\left(W^{u}(q)-q\right)}$ вытекает, что $q \in \operatorname{int} D_{q}$. Множество $S_{p q}=D_{p} \cup D_{q} \cup C_{p q}$ гомеоморфно $(n-1)$-мерной сфере, которую мы будем называть характеристической сферой, соответствующей связке $B_{p q}$. В силу теоремы о структуре произведения и непрерывной зависимости инвариантных многообразий от начальных условий $S_{p q}$ является цилиндрически вложенной в $M$ сферой. Отметим, что характеристическая сфера не определяется однозначно связкой.

Слоение Рэба. Слоение, каждый слой которого гомеоморфен (во внутренней топологии) плоскости, называется слоением Рэба.

Слоение называется трансверсально ориентируемым, если сушествует непрерывное векторное поле, трансверсальное слоям слоения. В противном случае слоение называется трансверсально неориентируемым.

\section{§2. Вспомогательные результаты}

Далее базисное множество коразмерности один мы будем считать для определенности растягивающимся аттрактором. Ясно, что все утверждения могут быть переформулированы для сжимающегося репеллера.

Идеи доказательств следующих двух лемм содержатся в работе [6]. Доказательства можно извлечь из доказательств лемм 2.1, 2.2 работы [10] (в двумерном случае), теоремы 1 работы [25] или теоремы 2.1 работы [15] (в многомерном случае).

ЛЕмма 2.1. Пусть $\Omega$-растягивающийся аттрактор коразмерности один А-диффеоморфизма $f: M^{3} \rightarrow M^{3}$ многообразия $M^{3}$. Предположим, что неустойчивые многообразия $W^{u}\left(m_{1}\right), W^{u}\left(m_{2}\right) \subset \Omega$ образуют 1- или 2-связку (т.е. не исключается возмозсность $W^{u}\left(m_{1}\right)=W^{u}\left(m_{2}\right)$ ), где $m_{1}, m_{2}$ - ассоииированные граничные периодические точки. Тогда существует гомеомор$\oint и з м$

$$
\varphi: W^{u}\left(m_{1}\right)-m_{1} \rightarrow W^{u}\left(m_{2}\right)-m_{2}
$$

со следующими свойствами:

а) для любой точки $x \in W^{u}\left(m_{1}\right)-m_{1}$ точка $\varphi(x) \in W^{u}\left(m_{2}\right)-m_{2}$ лежсит на устойчивом многообразии $W^{s}(x)$, и замкнутая дуга устойчивого многообразия $W^{s}(x)$ с кониевыми точками $x, \varphi(x)$ пересекается $c \Omega$ только в кониевых точках $x, \varphi(x)$;

b) если $m_{1}$ является периодической периода р (в этом случае $m_{2}$ такәсе имеет период $p$ ), то

$$
\left.\varphi \circ f^{p}\right|_{W^{u}\left(m_{1}\right)-m_{1}}=\left.f^{p} \circ \varphi\right|_{W^{u}\left(m_{1}\right)-m_{1}},
$$

т.е. отображсния $f^{p}, \varphi$ коммутируют на $W^{u}\left(m_{1}\right)-m_{1}$.

ЛЕмма 2.2. Гомеоморфизм ч продолжсается до гомеоморфизма

$$
\varphi: W^{u}\left(m_{1}\right) \cup W^{u}\left(m_{2}\right) \rightarrow W^{u}\left(m_{1}\right) \cup W^{u}\left(m_{2}\right),
$$

если положить $\varphi\left(m_{1}\right)=m_{2} u \varphi\left(m_{2}\right)=m_{1}$. 
ЛЕМма 2.3. Пусть растягивающийся аттрактор $\Omega$ коразмерности один имеет 1-связку $W^{u}\left(m_{0}\right)$, где $m_{0}$ - граничная периодическая точка. Тогда $\Omega$ неориентируем в смысле Гринеса. Именно существует дуга

$$
(x, y)^{s} \subset W^{s}(x), \quad x, y \in W^{u}\left(m_{0}\right)-m_{0}, \quad[x, y]^{s} \cap \Omega=\{x ; y\},
$$

устойчивого многообразия $W^{s}(x)$, которая пересекает $W^{u}\left(m_{0}\right)$ в концевьх точках $x$, у с разными индексами пересечения.

ДокАЗАтЕльство. Так как неустойчивое многообразие $W^{u}\left(m_{0}\right)$ образует 1-связку, то сушествует дуга

$$
(x, y)_{\varnothing}^{s}=(x, y)^{s} \subset W^{s}(x), \quad x, y \in W^{u}\left(m_{0}\right)-m_{0} .
$$

Предположим, что $(x, y)^{s}$ пересекает $W^{u}\left(m_{0}\right)$ в концевых точках $x, y$ с одинаковыми индексами пересечения. Возьмем диск $D \subset W^{u}\left(m_{0}\right)$, содержащий точки $x, y$, и его окрестность $U$, гомеоморфную 3 -мерному шару, в многообразии $M^{3}$, которая компонентой пересечения $U \cap W^{u}\left(m_{0}\right)$, содержащей $D$, делит окрестность $U$ на две области $U_{1}, U_{2}$. В силу предположения обе области $U_{1}, U_{2}$ пересекаются с дугой $(x, y)^{s}$. Но любое неустойчивое многообразие аттрактора $\Omega$ самопредельное. Поэтому $W^{u}\left(m_{0}\right)$ пересекает одну из областей $U_{1}, U_{2}$ в бесконечном множестве точек. Следовательно, $W^{u}\left(m_{0}\right)$ должно пересекать дугу $(x, y)^{s}$. Это противоречит равенству $(x, y)_{\varnothing}^{s}=(x, y)^{s}$.

Далее в этом параграфе мы доказьваем лемму о том, что растягивающийся аттрактор коразмерности один ориентируем в смысле Гринеса тогда и только тогда, когда все его связки имеют степень два. По существу этот результат есть следствие теории слоений коразмерности один и замечательной теоремы Плыкина о вложении устойчивых и неустойчивых многообразий базисного множества в пару слоений дополнительных размерностей, анонсированной в [15] (теорема об инвариантных слоениях). Поскольку полного доказательства этой теоремы пока не опубликовано, то для удобства читателя мы приведем доказательство нужного нам утверждения. Сперва докажем одну вспомогательную лемму.

Лемма 2.4. Не существует трансверсально неориентируемых $C^{1}$-слоений Рэба на замкнутых трехмерных многообразиях.

ДокАЗАТЕльство. Предположим противное. Пусть $\mathscr{F}-$ трансверсально неориентируемое слоение Рэба на замкнутом трехмерном многообразии $M^{3}$. В [26] показано, что если $\mathscr{F}$ имеет класс гладкости $C^{2}$, то многообразие $M^{3}$ является трехмерным тором $T^{3}$. Гладкость $C^{2}$ используется для получения на некоторой поверхности общего положения морсовских особенностей. Эта техника была усовершенствована до класса гладкости $C^{1}$ Френксом [5]. Аккуратное изложение этой техники, начиная с приведения в общее положение поверхностей относительно слоения, можно найти в примечании Д. В. Аносова в книге [27]. Таким образом, модифицируя в соответствующих местах доказательство из [26], мы можем вывести, что многообразие $M^{3}$ является трехмерным тором $T^{3}$. Ниже мы будем применять 
результаты теории слоений, доказанные для слоений гладкости $C^{2}$, но справедливые в силу приведенных рассуждений для слоений гладкости $C^{1}$.

Пусть $\pi: \mathbb{R}^{3} \rightarrow T^{3} \cong \mathbb{R}^{3} / \mathbb{Z}^{3}$ - универсальное накрытие и $\overline{\mathscr{F}}$ - накрьвающее слоение для $\mathscr{F}$ относительно $\pi$, где $\mathbb{Z}^{3}$ - группа накрывающих преобразований, изоморфная трехмерной целочисленной решетке. Покажем, что $\overline{\mathscr{F}}$ трансверсально ориентируемое. Предположим противное. Тогда существуют трансверсальная к $\overline{\mathscr{F}}$ дуга $A \bar{x} \bar{y}$ с конщевыми точками $\bar{x}, \bar{y}$ и слой $\bar{l}$ слоения $\overline{\mathscr{F}}$ такие, что индексы пересечения $A_{\bar{x}} \bar{y} \mathrm{c} \bar{l}$ в точках $\bar{x}, \bar{y}$ различны. Соединим точки $\bar{x}, \bar{y}$ произвольным путем в слое $\bar{l}$. Этот путь совместно с $A_{\bar{x}} \bar{y}$ образует замкнутую петлю $P$. Так как $P \subset \mathbb{R}^{3}$, то существует гладкая иммерсия $\psi: D^{2} \rightarrow \mathbb{R}^{3}$ такая, что $\psi\left(\partial D^{2}\right)=P$, где $D^{2}-$ двумерньй диск. Мальм шевелением приведем диск $\psi\left(D^{2}\right)$ в общее положение относительно слоения $\overline{\mathscr{F}}$ таким образом, чтобы на полученном диске, который мы обозначим снова через $\psi\left(D^{2}\right)$, слоение $\overline{\mathscr{F}}$ индуцировало одномерное слоение $\mathscr{F}{ }_{D^{2}}$ с конечным числом морсовских особенностей типа центр и седло. Так как индексы пересечения $A_{\bar{x}} \bar{y}$ с $\bar{l}$ в точках $\bar{x}, \bar{y}$ различны, то индекс замкнутой кривой $P$ относительно слоения $\mathscr{F}_{D^{2}}$ равен $1 / 2$ (см. [28]). С другой стороны, индекс морсовских особенностей типа центр и седло целый. Поэтому сумма индексов особенностей слоения $\mathscr{F}_{D^{2}}$ должна быть целым числом. Полученное противоречие доказывает трансверсальную ориентируемость слоения $\overline{\mathscr{F}}$.

Согласно сделанному предположению о неориентируемости слоения $\mathscr{F}$ сушествуют трансверсальная к $\mathscr{F}$ дуга $A_{x y}$ с концевьми точками $x, y$ и слой $l$ такие, что индексы пересечения $A_{x y} \mathrm{c} l$ в точках $x, y$ различны. Не уменшшая общности, можно считать, что $A_{x y}$ незамкнута и не имеет самопересечений. Тогда $\pi^{-1}\left(A_{x y}\right)$ состоит из счетного семейства дуг. Возьмем одну из них, скажем, $\bar{A} \bar{x} \bar{y}$ с концевыми точками $\bar{x}, \bar{y}$.

Пусть $\bar{l}(\bar{x}), \bar{l}(\bar{y})$ - слои слоения $\overline{\mathscr{F}}$, проходящие через точки $\bar{x}, \bar{y}$ соответственно. Так как $\overline{\mathscr{F}}$ трансверсально ориентируемое и все слои односвязны, то $\bar{l}(\bar{x})$ и $\bar{l}(\bar{y})$ различны, в противном случае сушествовала бы замкнутая трансверсаль слоения $\overline{\mathscr{F}}$, гомотопная нулю [29]. Каждьй из слоев $\bar{l}(\bar{x}), \bar{l}(\bar{y})$ разбивает пространство $\mathbb{R}^{3}$ на области $R_{\bar{x}}^{+}, R_{\bar{x}}^{-}$и $R_{\bar{y}}^{+}, R_{\bar{y}}^{-}$соответственно. Предположим для определенности, что $\bar{l}(\bar{x}) \subset R_{\bar{y}}^{-}$и $\bar{l}(\bar{y}) \subset R_{\bar{x}}^{+}$. Тогда $\bar{A}_{\bar{x}} \bar{y} \subset R_{\bar{y}}^{-} \cap R_{\bar{x}}^{+}$, поскольку дуга $\bar{A}_{\bar{x}} \bar{y}$ соединяет слои $\bar{l}(\bar{x}), \bar{l}(\bar{y})$ и в силу трансверсальности слоению $\overline{\mathscr{F}}$ не может пересекать каждый из слоев дважды.

Так как $\bar{l}(\bar{x})$ и $\bar{l}(\bar{y})$ накрывают один и тот же слой $l$ слоения $\mathscr{F}$, то на $\bar{l}(\bar{y})$ сушествует точка $\bar{y}_{1}$, конгруэнтная точке $\bar{x}$, т.е. имеется единственный элемент $\gamma \in \mathbb{Z}^{3}$ такой, что $\gamma(\bar{x})=\bar{y}_{1}$. Напомним, что индексы пересечения дуги $A_{x y}$ с $l$ в точках $x, y$ различны. Это означает, что локально $A_{x y}$ подходит к слою $l$ в точках $x, y$ с одной и той же стороны. Поэтому дуги $\bar{A}_{\bar{x}} \bar{y}$ и $\gamma\left(\bar{A}_{\bar{x}} \bar{y}\right)$ лежат в одной компоненте множества $\mathbb{R}^{3}-\bar{l}(\bar{y})$, т.е. в $R_{\bar{y}}^{-}$. Следовательно,

$$
\gamma\left(R_{\bar{x}}^{-}\right)=R_{\bar{y}}^{+}, \quad \gamma\left(R_{\bar{x}}^{+}\right)=R_{\bar{y}}^{-}
$$

$\mathrm{B}[30]$ доказано сушествование отображения $\bar{h}: \mathbb{R}^{3} \rightarrow \mathbb{R}^{3}$, которое является накрьваюшим для сохраняющего ориентацию гомотопного тождественному непрерывного отображения $h: T^{3} \rightarrow T^{3}$ и которое переводит слоение $\overline{\mathscr{F}}$ в линейное слоение $\bar{h}(\overline{\mathscr{F}})$. Так как $\bar{h}$ сохраняет ориентацию, то слои $\bar{h}(\bar{l}(\bar{y})), \bar{h}(\bar{l}(\bar{x}))$ разбивают $\mathbb{R}^{3}$ 
на области $\bar{h}\left(R_{\bar{y}}^{-}\right), \bar{h}\left(R_{\bar{y}}^{+}\right)$и $\bar{h}\left(R_{\bar{x}}^{-}\right), \bar{h}\left(R_{\bar{x}}^{+}\right)$соответственно, причем

$$
\bar{h}(\bar{l}(\bar{y})) \subset \bar{h}\left(R_{\bar{x}}^{+}\right), \quad \bar{h}(\bar{l}(\bar{x})) \subset \bar{h}\left(R_{\bar{y}}^{-}\right) .
$$

Отметим, что в [30] предполагалась трансверсальная ориентируемость слоения на $T^{3}$. Однако анализ доказательств показывает, что достаточно предполагать только трансверсальную ориентируемость слоения на $\mathbb{R}^{3}$.

Так как $\bar{h}$ является накрываюшим для гомотопного тождественному непрерывного отображения, то $\bar{h}$ коммутирует с $\gamma$. Следовательно,

$$
\gamma \circ \bar{h}\left(R_{\bar{x}}^{-}\right)=\bar{h} \circ \gamma\left(R_{\bar{x}}^{-}\right)=\bar{h}\left(R_{\bar{y}}^{+}\right)
$$

$\mathrm{C}$ другой стороны, $\gamma$ является целочисленным сдвигом пространства $\mathbb{R}^{3}$ и слоение $\bar{h}(\overline{\mathscr{F}})$ состоит из геометрических плоскостей. Поэтому из включений (2) вытекает равенство $\gamma \circ \bar{h}\left(R_{\bar{x}}^{-}\right)=\bar{h}\left(R_{\bar{y}}^{-}\right)$. Полученное противоречие доказывает лемму.

ЛЕмма 2.5. Пусть $\Omega$ - растягивающийся аттрактор коразмерности один структурно устойчивого диффеоморфизма $f: M^{3} \rightarrow M^{3}$ замкнутого трехмерного многообразия $M^{3}$. Тогда $\Omega$ ориентируем в смисле Гринеса тогда и только тогда, когда все его связки имеют степень два.

ДокаЗАТЕЛЬство. Если $\Omega$ ориентируем в смысле Гринеса, то в силу леммы 2.3 степень связок аттрактора $\Omega$ равна двум (напомним, что в размерности большей двух Плыкин [15] доказал, что растягивающийся аттрактор коразмерности один может иметь только 1- и 2-связки). Следовательно, осталось доказать обратное утверждение.

Пусть все связки аттрактора $\Omega$ имеют степень два. Так как $f$ структурно устойчивый, то характеристические сферы, соответствующие всем 2-связкам аттрактора $\Omega$, ограничивают в $M^{3}$ трехмерные шары и $\Omega C$-плотное [31]. Отсюда и из теоремы Плыкина об инвариантных слоениях [15] (которая в этой частной ситуации сомнений не вызывает) вытекает, что неустойчивые многообразия аттрактора $\Omega$ вкладываются в слоение Рэба $\mathscr{F}$ и, более того, существует одномерное слоение $\mathscr{F} \perp$, трансверсальное слоению $\mathscr{F}$, такое, что его слои совпадают с устойчивыми многообразиями точек из $\Omega$ вне шаров, ограниченных характеристическими сферами. В силу [32] неустойчивые многообразия аттрактора $\Omega$ образуют $C^{1}$-ламинацию, поэтому $\mathscr{F}$ также можно считать $C^{1}$-слоением.

Согласно [26] многообразие $M^{3}$ является трехмерным тором $T^{3}$. Из леммы следует, что $\mathscr{F}$ трансверсально ориентируемое. Поэтому любая дуга произвольного слоя слоения $\mathscr{F}^{\perp}$ пересекается с любым диском произвольного слоя слоения $\mathscr{F}$ во всех точках пересечения с одинаковыми индексами пересечения (если пересечение непусто). В шарах, ограниченных характеристическими сферами, нет точек аттрактора $\Omega$ [31]. Так как слои одномерного слоения $\mathscr{F}^{\perp}$ совпадают с устойчивыми многообразиями точек из $\Omega$ вне шаров, ограниченных характеристическими сферами, то трансверсальная ориентируемость $\mathscr{F}$ влечет ориентируемость в смысле Гринеса аттрактора $\Omega$. 


\section{§ 3. Доказательство основных теорем}

В этом параграфе доказываются основные результаты статьи.

Теорема 3.1. Пусть $f: M^{3} \rightarrow M^{3}-$-диффеооморфизм, который имеет растягивающийся аттрактор $\Omega$ коразмерности один. Если $\Omega$ содерәит 1-связки, то многообразие $M^{3}$ неориентируемое.

ДокАЗАТЕЛЬство. По условию существует неустойчивое многообразие $W^{u}\left(m_{0}\right) \subset \Omega$, где $m_{0}$ - граничная периодическая точка диффеоморфизма $f$, образуюшее 1-связку аттрактора $\Omega$, т.е. имеется открытая дуга одномерного устойчивого многообразия с концевыми точками на $W^{u}\left(m_{0}\right)$, лежащая в $M^{3}-\Omega$. Не уменьшая общности, можно считать, что $m_{0}$ является неподвижной точкой диффеоморфизма $f$.

В силу леммы 2.1 сушествует гомеоморфизм $\varphi: W^{u}\left(m_{0}\right)-m_{0} \rightarrow W^{u}\left(m_{0}\right)-m_{0}$ со следующими свойствами:

1) для любой точки $x \in W^{u}\left(m_{0}\right)-m_{0}$ имеем $\varphi(x) \in W^{s}(x) \cap\left(W^{u}\left(m_{0}\right)-m_{0}\right)$, более того, дуга $(x, \varphi(x))^{s} \subset W^{s}(x), x \in W^{u}\left(m_{0}\right)-m_{0}$, с концевыми точками $x, \varphi(x)$ пересекается с $\Omega$ только в концевых точках;

2) $\left.\varphi \circ f\right|_{W^{u}\left(m_{0}\right)-m_{0}}=\left.f \circ \varphi\right|_{W^{u}\left(m_{0}\right)-m_{0}}$;

3) $\varphi^{2}=\mathrm{id}$ и $\left.\varphi\right|_{W^{u}\left(m_{0}\right)-m_{0}}$ не имеет неподвижных точек.

Дальнейшее доказательство разобьем для удобства на несколько шагов.

Шаг 1. Для любой точки $x \in W^{u}\left(m_{0}\right)-m_{0}$ имеем $f^{n}(x) \rightarrow m_{0}$ и $\varphi \circ f^{n}(x) \rightarrow m_{0}$ при $n \rightarrow-\infty$. Более того, обе последовательности сходятся равномерно на любом компакте многообразия $W^{u}\left(m_{0}\right)$.

Действительно, так как ограничение $\left.f^{-1}\right|_{W^{u}\left(m_{0}\right)}$ есть структурно устойчивое отображение с единственной притягивающей гиперболической точкой $m_{0}$, то $f^{n}(x) \rightarrow m_{0}$ и $f^{n} \circ \varphi(x) \rightarrow m_{0}$ при $n \rightarrow-\infty$. Из равенства $\varphi \circ f^{n}(x)=f^{n} \circ \varphi(x)$ следует, что $\varphi \circ f^{n}(x) \rightarrow m_{0}$ при $n \rightarrow-\infty$. Так как отображение $\left.f^{-1}\right|_{W^{u}\left(m_{0}\right)}$ имеет компактную фундаментальную область, то отсюда вытекает утверждение о равномерности пределов.

Шаг 2. Гомеоморфизм $\varphi: W^{u}\left(m_{0}\right)-m_{0} \rightarrow W^{u}\left(m_{0}\right)-m_{0}$ можно гомеоморфно продолжить на $W^{u}\left(m_{0}\right)$, положив $\varphi\left(m_{0}\right)=m_{0}$.

Это вытекает из шага 1.

Зафиксируем точку $x_{0} \in W^{u}\left(m_{0}\right)$ и рассмотрим семейство замкнутых дисков $D_{\alpha} \subset W^{u}\left(m_{0}\right)-m_{0}$ (индекс $\alpha$ пробегает интервал $\left.[0,1]\right)$ таких, что

a) $x_{0} \in D_{0}, \varphi\left(x_{0}\right) \notin D_{0}$;

b) $D_{\alpha_{1}} \subset D_{\alpha_{2}}$, если $\alpha_{1}<\alpha_{2}$;

c) граница $\partial D_{\alpha}$ непрерывно деформируется при изменении $\alpha$;

d) $\varphi\left(x_{0}\right) \in D_{1}$.

Так как $W^{u}\left(m_{0}\right)$ гомеоморфно евклидовой плоскости $\mathbb{R}^{2}$ и $x_{0} \neq \varphi\left(x_{0}\right)$, то такое семейство дисков сушествует.

Шаг 3. Существует индекс $\alpha_{0} \in(0,1)$ такой, что

$$
\varnothing \neq D_{\alpha_{0}} \cap \varphi\left(D_{\alpha_{0}}\right)=\partial D_{\alpha_{0}} \cap \partial\left(\varphi\left(D_{\alpha_{0}}\right)\right)
$$


Действительно, так как $\varphi$ - гомеоморфизм, то $\varphi\left(D_{\alpha_{1}}\right) \subset \varphi\left(D_{\alpha_{2}}\right)$, если $\alpha_{1}<\alpha_{2}$. Из $\varphi\left(x_{0}\right) \notin D_{0}$ вытекает, что $D_{\alpha} \cap \varphi\left(D_{\alpha}\right)=\varnothing$ при малых $\alpha$. С другой стороны, из $\varphi\left(x_{0}\right) \in D_{1}$ следует $D_{\alpha_{1}} \cap \varphi\left(D_{\alpha_{1}}\right) \neq \varnothing$. Так как граница $\partial D_{\alpha}$ непрерывно деформируется при изменении $\alpha$ и $\varphi$ - гомеоморфизм, то граница $\partial \varphi\left(D_{\alpha}\right)=\varphi\left(\partial D_{\alpha}\right)$ также непрерывно деформируется при изменении $\alpha$. Отсюда вытекает требуемое утверждение.

Шаг 4. Сушествует кривая $C \subset W^{u}\left(m_{0}\right)-m_{0}$ без самопересечений с концевыми точками $x_{0}, \varphi\left(x_{0}\right)$ такая, что $C \cap \varphi(C)=\left\{x_{0}, \varphi\left(x_{0}\right)\right\}$.

В самом деле, согласно шагу 3 сушествует точка $x_{1} \in \partial D_{\alpha_{0}} \cap \varphi\left(\partial D_{\alpha_{0}}\right)$. В силу свойств гомеоморфизма $\varphi$ имеем

$$
\varphi\left(x_{1}\right) \in \partial D_{\alpha_{0}} \cap \varphi\left(\partial D_{\alpha_{0}}\right), \quad \varphi\left(x_{1}\right) \neq x_{1} .
$$

Так как $D_{\alpha_{0}}$ гомеоморфно диску, то сушествует путь $C_{01} \subset D_{\alpha_{0}}$ без самопересечений, соединяющий точки $x_{0}, x_{1}$, см. рис. 1 . Согласно шагу 3 внутренность диска $D_{\alpha_{0}}$ и ее образ относительно $\varphi$ не пересекаются. Поэтому $\varphi\left(C_{01}\right) \subset \varphi\left(D_{\alpha_{0}}\right)$ есть путь без самопересечений, который соединяет точки $\varphi\left(x_{0}\right), \varphi\left(x_{1}\right)$ и не пересекается с $C_{01}$. Так как кривая $\varphi\left(C_{01}\right)$ не разбивает диск $\varphi\left(D_{\alpha_{0}}\right)$, то существует путь $C_{12}$ без самопересечений, который соединяет точки $\varphi\left(x_{0}\right), x_{1}$ и не пересекается с путем $\varphi\left(C_{01}\right)$. В силу равенства $\varphi^{2}=\mathrm{id}$ объединение $C=C_{01} \cap C_{12}$ дает искомьй путь.

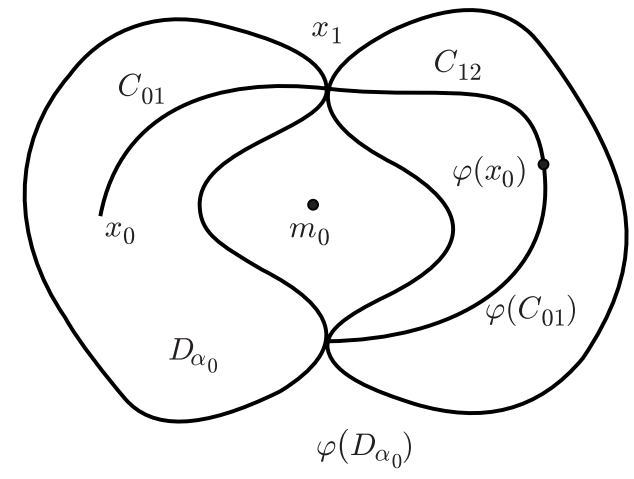

Рис. 1. Кривая $C=C_{01} \cup C_{12}$

Шаг 5. Множество $M_{c}=\bigcup_{x \in C}(x, y)_{\varnothing}^{s}$ гомеоморфно открытому листу Мёбиуса, при этом любая дуга $(x, y)_{\varnothing}^{s}$ трансверсальна (в топологическом смысле) средней линии этого листа Мёбиуса.

Докажем это. Рассмотрим открытьй прямоугольник $P=\bigcup_{x \in C-x_{0}}(x, y)_{\varnothing}^{s}$. Множество $M_{c}$ получается из прямоугольника $P$ добавлением дуги $\left(x_{0}, \varphi\left(x_{0}\right)\right)^{s}$. В силу равенства $\varphi^{2}=\mathrm{id}$ это добавление дуги можно представить как отождествление в замкнутом прямоугольнике $\operatorname{clos} P$ двух противоположных сторон с помощью обрашающего ориентацию гомеоморфизма. Отсюда следует, что $M_{c}$ гомеоморфно листу Мёбиуса. Из этой конструкции вытекает, что любая дуга $(x, y)_{\varnothing}^{s}$ трансверсальна (в топологическом смысле) средней линии этого листа Мёбиуса. 
В силу шага 4 объединение $C \cup \varphi(C)=C_{0}$ образует простую замкнутую кривую, ограничивающую на неустойчивом многообразии $W^{u}\left(m_{0}\right)$ диск, который мы обозначим через $D_{c}$.

Шаг 6. $m_{0} \in D_{c}$.

Действительно, внешность кривой $C_{0}$ на неустойчивом многообразии $W^{u}\left(m_{0}\right)$ гомеоморфна открытому проколотому цилиндру. Из $\varphi^{2}=\mathrm{id}$ вытекает $\varphi\left(C_{0}\right)=C_{0}$. Так как $\varphi$ - гомеоморфизм, то он должен переводить диск $D_{c}$ в себя (а не во внешность $)$. Тогда $\varphi\left(C_{0} \cup D_{c}\right)=C_{0} \cup D_{c}$, откуда следует наличие неподвижной точки у $\varphi$. В силу шага $2 m_{0}$ - единственная неподвижная точка гомеоморфизма $\varphi$.

Шаг 7. Сушествует открытое 3-мерное подмногообразие $C^{3} \subset M^{3}$, гомеоморффное прямому произведению $M_{b} \times(0,1)$, где $M_{b}$ - открытый лист Мёбиуса.

Докажем это. Воспользуемся предыдущими обозначениями. Возьмем простую открытую дугу $l_{0} \subset D_{c}$ с концевой точкой $x_{0}$ такую, что $l_{0} \cap \varphi\left(l_{0}\right)=\varnothing$. Такая дуга сушествует, поскольку $\varphi\left(x_{0}\right) \neq x_{0}$ и $\varphi$ - гомеоморфизм. Так как пересечение $\varphi(C) \cap C$ содержит только две точки $x_{0}, \varphi\left(x_{0}\right)$, то существует открытая полоса $P_{1} \subset D_{c}$, ограниченная кривой $\varphi(C)$ и дугами $l_{0}, \varphi\left(l_{0}\right)$, такая, что $\varphi\left(P_{1}\right) \cap P_{1}=\varnothing$. В силу шага $2 m_{0} \notin \varphi\left(P_{1}\right) \cup P_{1}$. Поэтому $\varphi\left(P_{1}\right)$ является открытой полосой, ограниченной кривой $C$ и дугами $l_{0}, \varphi\left(l_{0}\right)$. Следовательно, объединение $P=\varphi\left(l_{0}\right) \cup l_{0} \cup \varphi\left(P_{1}\right) \cup P_{1}$ также является открытой полосой в $D_{c}$, одна из граничных компонент которой есть граница диска $\partial D_{c}=\varphi(C) \cup C$.

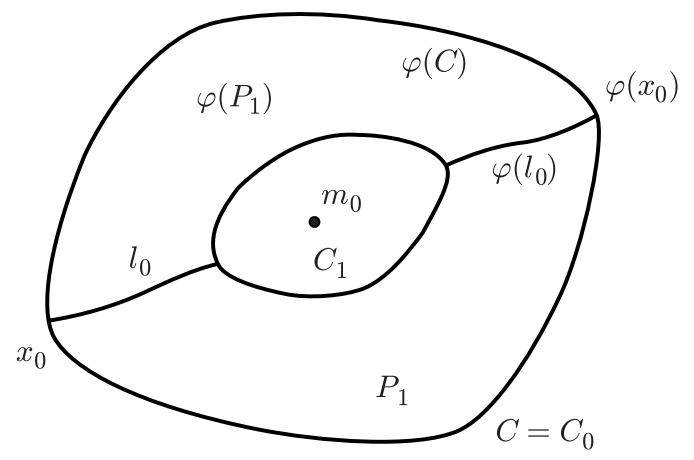

Рис. 2. Диск $D_{c}$ и полоса $P=P_{1} \cup \varphi\left(P_{1}\right) \cup l_{0} \cup \varphi\left(l_{0}\right)$

В границу полосы $P_{1} \subset D_{c}$ входят две противоположные дуги $l_{0}, \varphi\left(l_{0}\right)$ и вторая пара противоположных дуг, одна из которых есть кривая $C$. Противоположную $C$ дугу обозначим через $C_{1}$ и переобозначим $C=C_{0}$, см. рис. 2 . Заметим, что противоположные точки каждой из дуг $C_{1}$ и $C_{0}$ переходят друг в друга под действием $\varphi$. Поэтому существует расслоение замкнутой полосы $\varphi\left(l_{0}\right) \cup l_{0} \cup P_{1} \cup C_{0} \cup C_{1}$ на попарно непересекающиеся дуги $C_{\alpha}$ (индекс $\alpha$ пробегает все значения интервала $\left.[0,1]\right)$, концевые точки которых переходят друг в друга под действием $\varphi$. Тогда объединение $C_{\alpha} \cup \varphi\left(C_{\alpha}\right)$ является простой замкнутой кривой для любого $\alpha \in[0,1]$. Так как $\varphi$-гомеоморфизм, то отсюда вытекает, что семейство $C_{\alpha} \cup \varphi\left(C_{\alpha}\right), \alpha \in[0,1]$, образует расслоение полосы $P$ на попарно непересекающиеся концентрические окруж- 
ности, инвариантные относительно $\varphi$. Аналогично шагу 5 показывается, что каждая окружность $C_{\alpha} \cup \varphi\left(C_{\alpha}\right)$ является границей множества

$$
M_{\alpha}=\bigcup_{x \in C_{\alpha} \cup \varphi\left(C_{\alpha}\right)}(x, \varphi(x))^{s}
$$

гомеоморфного открытому листу Мёбиуса $M_{b}$. Так как устойчивые многообразия попарно не пересекаются, то множества $M_{\alpha}$ также попарно не пересекаются. Из непрерывной зависимости инвариантных многообразий от начальных условий на компактных подмножествах вытекает, что множества $M_{\alpha}$ непрерьвно зависят от $\alpha$. Отсюда и из гомеоморфности полосы $P$ прямому произведению $S^{1} \times(0,1)$ ( $S^{1}$ - окружность) вытекает, что открытое 3 -мерное многообразие

$$
C^{3}=\bigcup_{x \in P}(x, \varphi(x))^{s}=\bigcup_{\alpha \in(0,1)} M_{\alpha}
$$

гомеоморфно прямому произведению $M_{b} \times(0,1)$.

Так как лист Мёбиуса является неориентируемой поверхностью, то 3-мерное подмногообразие $C^{3}$ является неориентируемым 3-мерным многообразием [33]. Следовательно, $M^{3}$ - также неориентируемое 3 -мерное многообразие.

Непосредственно из доказательства теоремы 3.1 вытекает

СлЕДСТВИЕ 3.1. Пусть выполняются условия теоремы 3.1, и пусть $R$ открытая компонента области $M^{3}-\Omega$ такая, что ее достижимая граница содерэит неустойчивое многообразие $W^{u}\left(m_{0}\right) \subset \Omega$, образующее 1-связку, где $m_{0}$ - граничная периодическая точка диффеоморфизма $f$. Тогда существует открытое 3-мерное подмногообразие $C^{3} \subset R \cap\left(W^{s}(\Omega)-\Omega\right)$, гомеоморфное прямому произведению $M_{b} \times(0,1)$, где $M_{b}$ - открытый лист Мёбиуса. И более того, пересечение $\cos C \cap \Omega$ является компактным кольиом в $W^{u}\left(m_{0}\right)$.

СлЕДСТВИЕ 3.2. Пусть выполняются условия теоремы 3.1, и пусть $R$ открытая компонента области $M^{3}-\Omega$ такая, что ее достижимая граница содержит неустойчивое многообразие $W^{u}\left(m_{0}\right) \subset \Omega$, образующее 1-связку, где $m_{0}$ - граничная периодическая точка диффеоморфизма $f$. Тогда фундаментальная группа $\pi_{1}(R)$ нетривиальна.

ДокАЗАТЕльство. В силу следствия 3.1 существует открытое 3-мерное подмногообразие $C^{3} \subset R \cap\left(W^{s}(\Omega)-\Omega\right)$, гомеоморфное прямому произведению $M_{b} \times(0,1)$, где $M_{b}$ - открытый лист Мёбиуса. Поэтому $R$ является неориентируемьм открытым многообразием. Отсюда вытекает требуемый результат.

СледСТВИЕ 3.3. Если А-диффеоморфизм $f: M^{3} \rightarrow M^{3}$ имеет двумерный аттрактор Плыкина, то трехмерное замкнутое многообразие $M^{3}$ неориентируемое.

ТЕОРема 3.2. Пусть $\Omega$ - растягивающийся аттрактор или сжиммающийся репеллер коразмерности один структурно устойчивого диффеоморфизма $f: M^{3} \rightarrow M^{3}$ компактного многообразия $M^{3}$. Тогда $\Omega$ не имеет 1-связок. 
ДокАЗАТЕльство. Для определенности будем считать $\Omega$ аттрактором. Предположим, что теорема неверна. Тогда сушествует открытая компонента $R \subset$ $M^{3}-\Omega$, в достижимую границу которой входит 1 -связка $W^{u}\left(m_{0}\right) \subset \Omega$. Не уменьшая общности, можно считать точку $m_{0}$ неподвижной, перейдя, если необходимо, к некоторой степени $f^{k}$. В силу теоремы 3.1 многообразие $M^{3}$ неориентируемое. Пусть $\widetilde{M^{3}}$ - двулистное ориентируемое накрытие для $M^{3}$ и $p: \widetilde{M}^{3} \rightarrow M^{3}$ - проекция $\widetilde{M}^{3}$ на $M^{3}$. Тогда сушествует накрьвающий $f$ диффеоморфизм $\widetilde{f}: \widetilde{M}^{3} \rightarrow \widetilde{M}^{3}$. Покажем, что $\tilde{f}$ является $A$-диффеоморфизмом.

Действительно, очевидно, что неблуждаюшая точка диффеоморфизма $\widetilde{f}$ проектируется в неблуждающую точку диффеоморфизма $f$, поскольку $p$ - локальньй гомеоморфизм. Поэтому $N W(\widetilde{f}) \subset p^{-1}(N W(f))$, где $N W(\widetilde{f})$ означает неблуждающее множество преобразования $\widetilde{f}$. Так как $f-A$-диффеоморфизм, то в $N W(f)$ всюду плотны периодические точки. Из двулистности накрытия вытекает, что прообраз периодической орбиты состоит либо из одной, либо из двух периодических орбит. Следовательно, в $p^{-1}(N W(f))$ всюду плотны периодические точки и поэтому $p^{-1}(N W(f)) \subset N W(\widetilde{f})$. Отсюда $N W(\widetilde{f})=p^{-1}(N W(f))$. Из этого равенства и из гиперболичности $N W(f)$ вытекает гиперболичность множества $N W(\tilde{f})$. Следовательно, $\widetilde{f}-A$-диффеоморфизм.

Из замкнутости и инвариантности (относительно $f$ ) множества $\Omega$ вытекает замкнутость и инвариантность (относительно $\widetilde{f}$ ) множества $p^{-1}(\Omega)$. Если $O\left(x_{0}\right)-$ всюду плотная в $\Omega$ орбита точки $x_{0} \in \Omega$, то $p^{-1}\left(O\left(x_{0}\right)\right)$ всюду плотно в $p^{-1}(\Omega)$. Из двулистности накрытия следует, что $p^{-1}\left(x_{0}\right)$ состоит из двух точек $\widetilde{x}_{1}, \widetilde{x}_{2}$, орбиты $O\left(\widetilde{x}_{1}\right), O\left(\widetilde{x}_{2}\right)$ которых в совокупности всюду плотны в $p^{-1}(\Omega)$. Очевидно, каждое из множеств $\operatorname{clos} O\left(\widetilde{x}_{1}\right), \operatorname{clos} O\left(\widetilde{x}_{2}\right)$ замкнуто, инвариантно и содержит всюду плотную орбиту, т.е. является базисным множеством $A$-диффеоморфизма $\widetilde{f}$. Из равенства

$$
\operatorname{clos} O\left(\widetilde{x}_{1}\right) \cup \operatorname{clos} O\left(\widetilde{x}_{2}\right)=\operatorname{clos}\left(O\left(\widetilde{x}_{1}\right) \cup O\left(\widetilde{x}_{2}\right)\right)=p^{-1}(\Omega)
$$

и единственности спектрального разложения [7] получаем, что $p^{-1}(\Omega)$ либо является базисным множеством, либо представляет собой объединение двух базисных множеств $\widetilde{\Omega}_{1}, \widetilde{\Omega}_{2} A$-диффеоморфизма $\widetilde{f}$. Мы будем писать $\widetilde{\Omega}_{1} \cup \widetilde{\Omega}_{2}=p^{-1}(\Omega)$, не исключая возможности $\widetilde{\Omega}_{1}=\widetilde{\Omega}_{2}$ и иногда опуская индекс, если конкретный выбор базисного множества неважен.

Так как $\Omega$ локально гомеоморфно прямому произведению канторовского множества на плоскость, то $\widetilde{\Omega}$ также локально гомеоморфно прямому произведению канторовского множества на плоскость. Следовательно, $\widetilde{\Omega}$ является растягивающимся аттрактором коразмерности один. Из ориентируемости $\widetilde{M}^{3}$ и теоремы 3.1 вытекает, что $\widetilde{\Omega}$ не имеет 1-связок. Плыкин [15] доказал, что в размерности $\operatorname{dim} M^{n} \geqslant 3$ растягиваюшийся аттрактор может иметь только 1- и 2-связки. Следовательно, $\widetilde{\Omega}$ имеет только 2-связки.

Так как $p$ - локальный диффеоморфизм и $f$ удовлетворяет сильному условию трансверсальности, то $\tilde{f}$ также удовлетворяет сильному условию трансверсальности. Следовательно, диффеоморфизм $\widetilde{f}$ структурно устойчивый [23]. Возьмем произвольное поднятие $\widetilde{m}_{1} \in \widetilde{\Omega}$ точки $m_{0}$. Нетрудно видеть, что $\widetilde{m}_{1}$ является граничной периодической точкой аттрактора $\widetilde{\Omega}$. Так как $\widetilde{\Omega}$ имеет только 2 -связки, то 
существует ассоциированная с $\widetilde{m}_{1}$ граничная периодическая точка $\widetilde{m}_{2} \in \widetilde{\Omega}$, отличная от $\widetilde{m}_{1}$. Для структурно устойчивых диффеоморфизмов компактных $n$-мерных $(n=\operatorname{dim} M \geqslant 3)$ многообразий $M$ в работе [31] доказано, что $W_{\varnothing}^{s}\left(\widetilde{m}_{1}\right)$ и $W_{\varnothing}^{s}\left(\widetilde{m}_{2}\right)$ содержатся в неустойчивых многообразиях $W^{u}\left(\widetilde{\alpha}_{1}\right), W^{u}\left(\widetilde{\alpha}_{k+1}\right)(k \geqslant 0$ - целое число, уточняемое ниже) отталкивающих периодических точек $\widetilde{\alpha}_{1}, \widetilde{\alpha}_{k+1}$ соответственно, орбиты которых являются тривиальными репеллерами (т.е. имеют индекс 3). Более того, в области $R \subset M^{3}-\Omega$ имеются периодические точки $\widetilde{\alpha}_{1}, \ldots, \widetilde{\alpha}_{k+1}$ (соответственно $\left.P_{1}, \ldots, P_{k}\right)$ индекса 3 (соответственно индекса 2$)$, которые удовлетворяют следуюшим условиям:

1) множество

$a_{12}=\left\{\widetilde{m}_{1}\right\} \cup W_{\varnothing}^{s}\left(\widetilde{m}_{1}\right) \cup \widetilde{\alpha}_{1} \cup W^{s}\left(P_{1}\right) \cup P_{1} \cup \widetilde{\alpha}_{2} \cup \cdots \cup P_{k} \cup \widetilde{\alpha}_{k+1} \cup W_{\varnothing}^{s}\left(\widetilde{m}_{2}\right) \cup\left\{\widetilde{m}_{2}\right\}$

является простой компактной связной дугой с концевыми точками $\widetilde{m}_{1}$ и $\widetilde{m}_{2}$;

2 ) на дуге $a_{12}$ периодические точки $\widetilde{\alpha}_{i}$ индекса 3 чередуются с периодическими точками $P_{i}$ индекса 2 (включая точки $\widetilde{m}_{1}$ и $\widetilde{m}_{2}$ ), см. рис. 3.

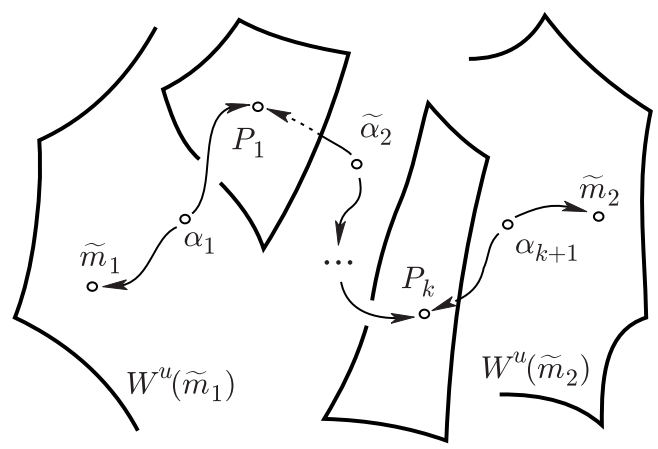

Рис. 3. Дуга $a_{12}$

В силу леммы 2.1 если $\widetilde{x}_{n} \rightarrow \widetilde{m}_{1}$ при $n \rightarrow \infty$, то $\widetilde{y}_{n} \rightarrow \widetilde{m}_{2}$ при $n \rightarrow \infty$, где $\widetilde{x}_{n} \in W^{s}\left(\widetilde{m}_{1}\right), \widetilde{y}_{n} \in W^{u}\left(\widetilde{m}_{2}\right)$, и $\left(\widetilde{x}_{n}, \widetilde{y}_{n}\right)^{s}=\left(\widetilde{x}_{n}, \widetilde{y}_{n}\right)_{\varnothing}^{s}$ (напомним, что последнее равенство означает, что $\left.\left(\widetilde{x}_{n}, \widetilde{y}_{n}\right)^{s} \cup \widetilde{\Omega}=\varnothing\right)$. Поскольку накрытие $p$ осушествляет полусопряженность между $\widetilde{f}$ и $f$, то $p$ переводит инвариантные многообразия диффеоморфизма $\widetilde{f}$ в инвариантные многообразия диффеоморфизма $f$. Отсюда и из равенства $p(\widetilde{\Omega})=\Omega$ вытекает равенство

$$
p\left(\left(\widetilde{x}_{n}, \widetilde{y}_{n}\right)_{\varnothing}^{s}\right)=\left(p\left(\widetilde{x}_{n}\right), p\left(\widetilde{y}_{n}\right)\right)_{\varnothing}^{s}
$$

Так как $p\left(\widetilde{x}_{n}\right) \in W^{u}\left(m_{0}\right)$, то $p\left(\widetilde{y}_{n}\right) \in W^{u}\left(m_{0}\right)$, поскольку неустойчивое многообразие $W^{u}\left(m_{0}\right)$ образует 1-связку. Из непрерьвности $p$ получаем, что $p\left(\widetilde{x}_{n}\right) \rightarrow$ $p\left(\widetilde{m}_{1}\right)=m_{0}$ и $p\left(\widetilde{y}_{n}\right) \rightarrow p\left(\widetilde{m}_{2}\right)$. Из включения $p\left(\widetilde{y}_{n}\right) \in W^{u}\left(m_{0}\right)$ и леммы 2.2 вытекает, что $p\left(\widetilde{y}_{n}\right) \rightarrow m_{0}$. Следовательно, $p\left(\widetilde{m}_{2}\right)=m_{0}$. Таким образом, концевые 
точки дуги $a_{12}$ отображаются под действием $p$ в одну точку. Поскольку $p$ переводит инвариантные многообразия диффеоморфизма $\widetilde{f}$ в инвариантные многообразия диффеоморфизма $f$, то

$$
\begin{gathered}
p\left(W_{\varnothing}^{s}\left(\widetilde{m}_{1}\right)\right)=p\left(W_{\varnothing}^{s}\left(\widetilde{m}_{2}\right)\right), \quad p\left(\widetilde{\alpha}_{1}\right)=p\left(\widetilde{\alpha}_{k+1}\right) \\
p\left(W^{s}\left(P_{1}\right)\right)=p\left(W^{s}\left(P_{k}\right)\right), \quad p\left(P_{1}\right)=p\left(P_{k}\right), \quad p\left(\widetilde{\alpha}_{2}\right)=p\left(\widetilde{\alpha}_{k}\right), \ldots .
\end{gathered}
$$

На дуге $a_{12}$ периодические точки индекса 2 чередуются с периодическими точками индекса 3 . Так как на концах дуги $a_{12}$ расположены периодические точки индекса 3 , то число периодических точек на $a_{12}$ нечетно. Следовательно, существует либо периодическая точка $\widetilde{\alpha}_{i}$ индекса 3 с $p\left(W^{s}\left(P_{i-1}\right)\right)=p\left(W^{s}\left(P_{i}\right)\right)$, либо периодическая точка $P_{i}$ индекса 2 такая, что обе компоненты множества $W^{s}\left(P_{i}\right)-P_{i}$ отображаются под действием $p$ в одну компоненту множества $W^{s}\left(p\left(P_{i}\right)\right)-p\left(P_{i}\right)$. В обоих случаях имеется точка $\left(\widetilde{\alpha}_{i}\right.$ либо $\left.P_{i}\right)$, в окрестности которой $p$ не является локальньм гомеоморфизмом. Полученное противоречие доказывает теорему.

СЛЕДСТВИЕ 3.4. Пусть $f: M^{3} \rightarrow M^{3}$ - структурно устойчивый диффеоморфизм замкнутого трехмерного многообразия $M^{3}$. Тогда $f$ не имеет двумерных базисных множеств Плыкина.

Теорема 3.3. Пусть $f: M^{3} \rightarrow M^{3}$ - структурно устойчивый диффеоморфизм замкнутого трехмерного многообразия $M^{3}$. Тогда спектральное разложсение диффеоморфизма $f$ не содержит неориентируемых растягивающихся аттракторов и сәмимающихся репеллеров коразмерности один.

ДокАЗАТЕЛЬСтво. Пусть $\Omega$ - растягивающийся аттрактор коразмерности один диффеоморфизма $f$. Тогда в силу теоремы $3.2 \Omega$ не имеет 1-связок. Из леммы 2.5 следует, что $\Omega$ ориентируем в смысле Гринеса.

СЛЕДСТВИЕ 3.5. Пусть $f: M^{3} \rightarrow M^{3}$ - структурно устойчивый диффеоморфизм замкнутого трехмерного многообразия $M^{3}$, имеющий растягивающийся аттрактор или сжимающийся репеллер коразмерности один. Тогда $M^{3}$ является трехмерныц тором $T^{3}$.

ДокАЗАТЕЛЬСтво. В [31] доказано, что если $f: M^{3} \rightarrow M^{3}$-структурно устойчивый диффеоморфизм замкнутого трехмерного многообразия $M^{3}$, имеющий ориентируемый растягивающийся аттрактор или сжимающийся репеллер коразмерности один, то $M^{3}$ является трехмерным тором $T^{3}$. Отсюда и из теоремы 3.3 вытекает требуемый результат.

Пример базисного множества Плыкина коразмерности один на замкнутом 3-многообразии. После теоремы 3.3 естественно рассмотреть вопрос о сушествовании неориентируемых растягивающихся аттракторов и сжимающихся репеллеров коразмерности один на замкнутых 3-многообразиях (у структурно неустойчивых в силу теоремы 3.3 диффеоморфизмов) и, в частности, базисных множеств Плыкина. Построим такой пример, следуя в идейном плане работе [34], где 
построен $A$-диффеоморфизм замкнутого 3 -многообразия с ориентируемыми растягивающимся аттрактором и сжимаюшимся репеллером коразмерности один. Основой построения будет пример Плыкина [15; теорема 2.3] на открытом 3-многообразии $M_{1}$, которое двулистно накрьвается 3 -тором $T^{3}$ с конечным числом вьколотых точек. Отправной точкой построения в [15] является $D A$-диффеоморфизм $f_{0}: T^{3} \rightarrow T^{3}$, коммутирующий с некоторой инволюцией $\theta: T^{3} \rightarrow T^{3}$. Для определенности будем считать, что нетривиальное базисное множество диффеоморфизма $f_{0}$ есть ориентируемый растягивающийся аттрактор $\Omega_{0}$. Тогда $f_{0}$ индуцирует $A$-диффеоморфизм $f_{1}: M_{1} \rightarrow M_{1}$ с неориентируемым растягивающимся аттрактором коразмерности один $\Omega_{1} \cong \Omega_{0} / \sim$, где $M_{1}=\left(T^{3}-\right.$ Fix $\left.\theta\right) / \sim$ - факторпространство по отношению эквивалентности “ ", отождествляющее точки $x, \theta(x)$. Через Fix $\theta$ обозначено множество неподвижных точек инволюции $\theta$. Естественная проекция $p: T^{3}-\operatorname{Fix} \theta \rightarrow M_{1}$ является двулистным накрытием.

Пусть $R_{1}$ - компонента множества $M_{1}-\Omega_{1}$, достижимая (из области $M_{1}-\Omega_{1}$ ) граница которой состоит из 1-связки $b_{1}$. Согласно [15; лемма 2.2] $R_{1}$ двулистно накрьвается областью $R_{0}$ с достижимой изнутри границей, состоящей из 2-связки $b_{0}=p^{-1}\left(b_{1}\right)$. Область $R_{0}$ гомеоморфна 3 -клетке (т.е. $\mathbb{R}^{3}$ ), из которой выколота одна точка $s_{0}$ - источниковая периодическая точка диффеоморфизма $f_{0}$. Эта точка $s_{0}$ является неподвижной точкой инволюции $\theta$. Поэтому область $R_{1} \cong R_{0} / \sim$ гомеоморфна $P^{2} \times \mathbb{R}^{1}$, где $P^{2}$ - проективная плоскость. Не уменьшая общности, можно считать $s_{0}$ также неподвижной точкой диффеоморфизма $f_{0}$.

Возьмем локальные координаты $\left(u_{1}, u_{2}, u_{3}\right): U \rightarrow \mathbb{R}^{3}$ в некоторой окрестности $U$ точки $s_{0}$ такие, что в этих координатах диффеоморфизм $f_{0}$ имеет вид

$$
f_{0}(\vec{u})=\lambda \vec{u}, \text { где } \vec{u}=\left(u_{1}, u_{2}, u_{3}\right) \in U, \quad \lambda>0 \text { - некоторое число. }
$$

Согласно теореме Гробмана-Хартмана [19] такая система координат существует. Фундаментальная (другое название - порождаюшая) область $D_{0}$ диффеоморфизма $\left.f_{0}\right|_{R_{0}}$ в координатах $\left(u_{1}, u_{2}, u_{3}\right)$ может быть взята как шаровое кольцо $1 / \sqrt{\lambda} \leqslant$ $|\vec{u}| \leqslant \sqrt{\lambda}$. Согласно построению Плькина [15] инволюция $\theta$ в окрестности $U$ действует как центральная симметрия. Поэтому фундаментальная область $D_{1}=$ $p\left(D_{0}\right) \cong D_{0} / \sim$ диффеоморфизма $\left.f_{1}\right|_{R_{1}}$ гомеоморфна $P^{2} \times[1 / \sqrt{\lambda}, \sqrt{\lambda}] \subset P^{2} \times \mathbb{R}^{1}$. Обозначим через $B_{1} \subset M_{1}$ область, гомеоморфную $P^{2} \times(-\infty, 1 / \sqrt{\lambda}) \subset P^{2} \times \mathbb{R}^{1}$. Отметим, что $B_{1}$ есть образ относительно проекции $p$ проколотого шара в $T^{3}-s_{0}$, ограниченного сферой $|u|=1 / \sqrt{\lambda}$.

Пусть $\psi_{0}: D_{0} \rightarrow D_{0}$ - гомеоморфизм вида

$$
\vec{u} \rightarrow \frac{\vec{u}}{|\vec{u}|^{2}}, \quad \frac{1}{\sqrt{\lambda}} \leqslant|\vec{u}| \leqslant \sqrt{\lambda} .
$$

Нетрудно проверить, что выполняется соотношение

$$
\left.\psi_{0} \circ f_{0}\right|_{|\vec{u}|=1 / \sqrt{\lambda}}=\left.f_{0}^{-1} \circ \psi_{0}\right|_{|\vec{u}|=1 / \sqrt{\lambda}} .
$$

Так как $\theta$ в окрестности $U$ действует как центральная симметрия, то $\psi_{0}$ индуцирует гомеоморфизм $\psi_{1}: D_{1} \rightarrow D_{1}$, которьй в силу (3) удовлетворяет соотношению

$$
\left.\psi_{1} \circ f_{1}\right|_{P^{2} \times\{0\}}=\left.f_{1}^{-1} \circ \psi_{1}\right|_{P^{2} \times\{0\}} .
$$


Пусть $f_{2}: M_{2} \rightarrow M_{2}$ - копия диффеоморфизма $f_{1}$ с соответствуюшими переобозначенными атрибутами (индекс 1 заменяется индексом 2) такими, как фундаментальная область и т. д. В частности, фундаментальная область $D_{2}$ также гомеоморфна $P^{2} \times[1 / \sqrt{\lambda}, \sqrt{\lambda}] \subset P^{2} \times \mathbb{R}^{1}$. Поэтому сушествует гомеоморфизм $\psi_{21}: D_{2} \rightarrow D_{1}$, аналогичный $\psi_{1}$ (и, следовательно, $\psi_{2}$ ). Отсюда вытекает, что $\psi_{21}$ в силу (4) удовлетворяет соотношениям

$$
\left.\psi_{21} \circ f_{2}^{-1}\right|_{P^{2} \times\{1\}}=\left.f_{1} \circ \psi_{21}\right|_{P^{2} \times\{1\}},\left.\quad \psi_{21} \circ f_{2}\right|_{P^{2} \times\{0\}}=\left.f_{1}^{-1} \circ \psi_{21}\right|_{P^{2} \times\{0\}} .
$$

Положим $x_{1} \approx x_{2}$, если $\psi_{21}\left(x_{2}\right)=x_{1}$. Определим многообразие $M_{12}^{3}$ как

$$
M_{12}^{3}=\left(M_{1}-B_{1}\right) \cup\left(M_{2}-B_{2}\right) / \approx .
$$

Диффеоморфизмы $f_{1}, f_{2}$ определяют диффеоморфизм $f_{12}: M_{12}^{3} \rightarrow M_{12}^{3}$ следуюшим образом:

$$
\begin{aligned}
& f_{12}(x)=f_{1}(x), f_{12}^{-1}(x)=f_{1}^{-1}(x), \quad \text { если } \quad x \in M_{1}-\operatorname{int}\left(B_{1} \cup D_{1}\right), \\
& f_{12}(x)=f_{2}(x), f_{12}^{-1}(x)=f_{2}(x), \quad \text { если } \quad x \in M_{2}-\operatorname{int}\left(B_{2} \cup D_{2}\right) .
\end{aligned}
$$

Для точки

$$
x \in\left(M_{1}-B_{1}\right) \cap\left(M_{2}-B_{2}\right)=\left(D_{1} \cup D_{2}\right) / \approx
$$

обозначим через $x_{i}$ представитель точки $x$ в $D_{i}(i=1,2)$, т.е. $x_{1} \approx x_{2}, x_{i} \in D_{i}$. Положим $f_{12}(x)=f_{1}\left(x_{1}\right)$ и $f_{12}^{-1}(x)=f_{2}\left(x_{2}\right)$. Для точек $x_{1} \in \cos B_{1} \cap D_{1}$, $x_{2}=\psi_{21}^{-1}\left(x_{1}\right) \approx x_{1}$ имеем в силу $(5)$

$$
\psi_{21} \circ f_{2}^{-1}\left(x_{2}\right)=f_{1} \circ \psi_{21}\left(x_{2}\right)=f_{1}\left(x_{1}\right) .
$$

Поэтому $f_{1}\left(x_{1}\right) \approx f_{2}^{-1}\left(x_{2}\right)$ и, следовательно, $f_{12}$ определен корректно.

Проделав аналогичное построение для всех 1-связок аттрактора $\Omega_{1}$ и соответствующих 1-связок $\Omega_{2}$, получим требуемый $A$-диффеоморфизм $f: M^{3} \rightarrow M^{3}$ замкнутого 3 -многообразия $M^{3}$ в себя, который имеет неориентируемый растягивающийся аттрактор $\Omega_{1}$ и сжимающийся репеллер $\Omega_{2}$ коразмерности один. Это завершает построение примера.

Отметим, что построенный диффеоморфизм $f$ имеет ровно два базисных множества $\Omega_{1}$ и $\Omega_{2}$. Так как $\Omega_{1}$ - аттрактор, а $\Omega_{2}$ - репеллер коразмерности один, то $f-\Omega$-устойчивый диффеоморфизм (см. [35; теорема В]).

ТЕОРема 3.4. Пусть $\Omega$ - растягивающийся аттрактор коразмерности один $A$-диффеоморфизма $f: M^{n} \rightarrow M^{n}$ компактного многообразия $M^{n}$ размерности $n \geqslant 3$. Тогда каждая компонента множества $M^{n}-\Omega$, в достижимую границу которой входит 1-связка, имеет нетривиальную фундаментальную группу. 
ДокАЗАТЕльство. В силу следствия 3.2 теорема верна для размерности $n=3$. Поэтому далее будем считать, что $n \geqslant 4$. Предположим, что теорема неверна. Тогда существует компонента $R \subset M^{n}-\Omega$, в достижимую границу которой входит 1-связка $W^{u}\left(m_{0}\right)$ такая, что фундаментальная группа $\pi_{1}(R)$ тривиальна. Так как множество $\Omega$ компактно, то одномерное подрасслоение $E_{\Omega}^{s}$ продолжается на все многообразие $M^{n}$ до поля линейных элементов $F$ с конечным числом особенностей.

Докажем сначала, что поле $F$ неориентируемо (т.е. не может быть наделено ориентацией, превращающей $F$ в векторное поле). Действительно, так как имеется 1-связка $W^{u}\left(m_{0}\right)$, то сушествует дуга

$$
[x, y]^{s}=[x, y]_{\varnothing}^{s}, \quad x, y \in W^{u}\left(m_{0}\right)-m_{0}
$$

устойчивого многообразия $W^{s}(x)$, которая пересекает $W^{u}\left(m_{0}\right)$ в концевых точках $x, y$ с разными индексами пересечения (см. лемму 2.3). Из определения устойчивых многообразий следует, что существует натуральное $n_{0}$ такое, что $f^{n_{0}}\left([x, y]^{s}\right)$ лежит в окрестности аттрактора $\Omega$ со структурой произведения [7]. Увеличивая $n_{0}$, можно окрестность со структурой произведения взять сколь угодно малой. Так как $F$ совпадает с $E_{\Omega}^{s}$ на $\Omega$, то в такой окрестности поле $F$ “близко" к подрасслоению $E_{\Omega}^{s}$. Из того, что дуга $f^{n_{0}}\left([x, y]^{s}\right)$ пересекает $W^{u}\left(m_{0}\right)$ в концевых точках с разньми индексами пересечения, вытекает, что $F$ неориентируемо, поскольку в противном случае $F$ было бы “сонаправлено" в достаточно малых окрестностях.

Согласно [36] существует двулистное неразветвленное накрытие $p: \bar{M}^{n} \rightarrow M^{n}$ такое, что поле линейных элементов $\bar{F}$, накрывающее $F$, ориентируемо. Обозначим через $R_{0}$ область $R$, из которой удалены особенности поля $F$. Так как $n \geqslant 4$, то фундаментальная группа области $R_{0}$ остается тривиальной. Поэтому полный прообраз $p^{-1}\left(R_{0}\right)$ состоит из двух связных компонент $\bar{R}_{1}, \bar{R}_{2}$ [36]. Из локальной гомеоморфности накрытия $p$ вытекает, что в достижимую границу каждой компоненты, скажем $\bar{R}_{1}$, входит слой $\bar{W} \subset p^{-1}(\Omega)$ коразмерности один, трансверсальньй полю $\bar{F}$. С другой стороны, вновь из локальной гомеоморфности $р$ следует, что имеется дуга из полного прообраза $p^{-1}\left(f^{n_{0}}\left([x, y]^{s}\right)\right)$, которая пересекает слой $\bar{W}$ в концевых точках с разными индексами пересечения и лежит в окрестности некоторой точки множества $p^{-1}(\Omega)$, которая может быть сколь угодно малой. Поэтому существуют сколь угодно малые окрестности, в которых векторы поля $\bar{F}$ "контрнаправлены". Это противоречит ориентируемости $\bar{F}$. Полученное противоречие доказывает теорему.

\section{Список литературы}

1. Аносов Д. В. Геодезические потоки на замкнутых римановых многообразиях отрицательной кривизны // Труды МИАН. 1967. Т. 90.

2. Аносов Д. В. Об одном классе инвариантных множеств гладких динамических систем // Труды пятой международной конференции по нелинейньм колебаниям. Т. 2. Качественные методы. Ин-т математики АН УССР. Киев: Наукова Думка, 1970. С. 39-45.

3. Аносов Д. В. Грубые системы // Труды МИАН. 1985. Т. 169. С. 59-93.

4. Аносов Д. В., Солодов В.В. Гиперболические множества // Итоги науки и техники. Соврем. пробл. матем. Фундам. напр. Динамические системы-9. Т. 66. М.: ВИНИТИ, 1991. C. $12-99$. 
5. Franks J. Anosov diffeomorphisms // Global analysis. Proc. Sympos. Pure Math. 1970. V. 14. P. 61-94. (пер.: // Гладкие динамические системы. М.: Мир, 1977. С. 32-86.)

6. Newhouse S. On codimension one Anosov diffeomorphisms // Amer. J. Math. 1970. V. 92. № 3. Р. 761-770. (пер.: // Гладкие динамические системы. М.: Мир, 1977. С. 87-98.)

7. Smale S. Differentiable dynamical systems // Bull. Amer. Math. Soc. (N.S.). 1967. V. 73. №1. P. 741-817. (пер.: // УМH. 1970. Т. 25. С. 113-185.)

8. Kollmer H. On hyperbolic attractors of codimension one // Lecture Notes in Math. 1977. V. 597. P. 330-334.

9. Plante J. The homology class of an expanded invariant manifolds // Lecture Notes in Math. 1975. V. 468. P. 251-256.

10. Гринес В.З. О топологической сопряженности диффеоморфизмов двумерного многообразия на одномерных ориентируемых базисных множествах. I // Труды ММО. 1975. T. 32. С. 35-60; II // Труды ММО. 1977. Т. 34. С. 234-252.

11. Плькин Р. В. Источники и стоки $A$-диффеоморфизмов поверхностей // Матем. сб. 1974. T. 94. C. $243-264$.

12. Гринес В.З., Калай X. Х. Диффеоморфизмы двумерных многообразий с просторно расположенньми базисными множествами // УМН. 1985. Т. 40. № 1. С. 189-190.

13. Плькин Р. В. О существовании притягивающих (отталкивающих) периодических точек $A$-диффеоморфизмов проективной плоскости и бутылки Клейна // УМН. 1977. Т. 32. №3. C. 179 .

14. Плькин Р. В. О гиперболических аттракторах диффеоморфизмов // УМН. 1980. Т. 35. №3. C. $94-104$.

15. Плыкин Р. В. О геометрии гиперболических аттракторов гладких каскадов // УМН. 1984. T. 39. №6. C. $75-113$.

16. Жиров А. Ю. Перечисление гиперболических аттракторов на ориентируемых поверхностях и применения к псевдоаносовским гомеоморфизмам // Докл. РАН. 1993. Т. 330. №6. С. $683-686$.

17. Жиров А. Ю. Гиперболические аттракторы диффеоморфизмов ориентируемых поверхностей. І. Кодирование, классификация и накрытия // Матем. сб. 1994. Т. 185. №6. C. 3-50; II. Перечисление и применение к псевдоаносовским диффеоморфизмам // Матем. сб. 1994. Т. 185. №9. С. 29-80; III. Алгоритм классифоикации // Матем. сб. 1995. T. 186. №9. C. 59-82.

18. Плыкин Р. В. О топологии базисных множеств диффееоморфизмов Смейла // Матем. сб. 1971. T. 84. C. 301-312.

19. Katok A., Hasselblatt B. Introduction to the modern theory of dynamical systems. Cambridge: Cambridge Univ. Press, 1997. (Encyclopedia Math. Appl. V. 54.)

20. Арансон C.X., Гринес В.З. Каскады на поверхностях // Итоги науки и техники. Соврем. пробл. матем. Фундам. напр. Динамические системы-9. Т. 66. М.: ВИНИТИ, 1991. C. $148-187$.

21. Гринес В.З. О топологической сопряженности диффеоморфизмов двумерного многообразия на одномерных базисных множествах // УМН. 1974. Т. 29. №6. С. 163-164.

22. Mañé R. A proof of $C^{1}$ stability conjecture // Publ. Math. Inst. Hautes Étud. Sci. 1988. V. 66. P. $161-210$.

23. Robinson C. Structural stability of $C^{1}$ diffeomorphisms // J. Differential Equations. 1976. V. 22. № 1. P. 28-73.

24. Келдьи Л. В. Топологические вложения в евклидово пространство // Труды МИАН. 1966. T. 81.

25. Гринес В.З., Жужома Е.В. О топологической классификации ориентируемых аттракторов на $n$-мерном торе // УМН. 1979. Т. 34. № 4. С. 185-186.

26. Rosenberg H. Foliations by planes // Topology. 1968. V. 7. P. 131-138.

27. Тамура И. Топология слоений. М.: Мир, 1979.

28. Aranson S., Belitsky G., Zhuzhoma E. Introduction to the qualitative theory of dynamical systems on surfaces. Providence, RI: Amer. Math. Soc., 1996. (Transl. Math. Monogr. V. 153.)

29. Новиков С. П. Топология слоений // Труды ММО. 1965. Т. 14. С. 248-278. 
30. Арансон C.X., Жужсома E.В. О топологической классификации слоений Риба коразмерности один на трехмерном торе // Методы качественной теории дифференциальных уравнений: Межвуз. матем. сб. / ред. Е.А. Леонтович-Андронова. Горький, 1978. C. $41-64$.

31. Grines V., Zhuzhoma E. Structuraly stable diffeomorphisms with codimension one basic sets // Preprint № 223. Université de Bourgogne, Laboratoire de topologie, Dijon Cedex, 2000 .

32. Hirsch M., Pugh C. Stablemanifolds and hyperbolic sets // Global analysis. Proc. Sympos. Pure Math. 1970. V. 14. P. 133-163.

33. Hempel J. 3-manifolds. Princeton, NJ: Princeton Univ. Press and Univ. Tokyo Press, 1976. (Ann. of Math. Stud. V. 86.)

34. Franks J., Robinson C. A quasi-Anosov diffeomorphism that is not Anosov // Trans. Amer. Math. Soc. 1976. V. 223. P. 267-278.

35. de Melo W. Structural stability of diffeomorphisms on two-manifolds // Invent. Math. 1973. V. 21. P. 233-246.

36. Спеньер Э. Алгебраическая топология. М.: Мир, 1971.

Нижегородский государственный технический университет;

Научно-исследовательский институт прикладной математики и кибернетики при Нижегородском государственном университете им. Н.И. Лобачевского
Поступила в редакцию 06.06.2001 